Review article

Paediatrics Today 2016;12(1):41-48

DOI $10.5457 / \mathrm{p} 2005-114.134$

\title{
ULTRASOUND ELASTOGRAPHY IN THE NONINVASIVE DIAGNOSIS OF LIVER DISEASE IN CHILDREN: A REVIEW
}

\author{
Ingy H. SLEMAN, Mark C. LISZEWSKI*
}

Department of Radiology, Division of Pediatric Radiology, Montefiore Medical Center and Albert Einstein College of Medicine, Bronx, NY, USA

$$
\begin{aligned}
& \text { *Corresponding author: } \\
& \text { markcliszewski@gmail.com } \\
& \text { Tel.: + 718-920-7748 } \\
& \text { Fax.: + 718-798-7983 }
\end{aligned}
$$

Received: October 30, 2015

Accepted: December 7, 2015

Key words: Ultrasound elastography Pediatric chronic liver disease - Hepatic fibrosis - Transient elastography - Shear wave.

\begin{abstract}
The objective of this review is to examine the clinical utility of ultrasound elastography (UE) in the work up of chronic pediatric liver disease as described in recent literature. We will discuss the technical aspects of the various UE techniques and review the elastography findings in several specific etiologies of chronic pediatric liver disease. These include non-alcoholic fatty liver disease, chronic viral hepatitis, cystic fibrosis, biliary atresia and liver transplant. Lastly, we will discuss the future direction of UE and unanswered questions that require further investigation before UE can be incorporated into routine clinical practice. Conclusion - Research over the past several years has shown ultrasound elastography to be a potentially useful tool in assessing extent, progression and prognosis of chronic pediatric liver disease. There are many potential clinical indications for the use of elastography in the evaluation of pediatric liver disease. Familiarity with this new non-invasive technique will be imperative in upcoming years as it moves from research into clinical practice.
\end{abstract}

\section{Introduction}

Chronic liver disease is a cause of significant morbidity and mortality worldwide. Chronic liver disease can progress to liver fibrosis and ultimately cirrhosis, which has potential to cause portal hypertension, hepatic insufficiency, and hepatocellular carcinoma (HCC). Staging of liver fibrosis is an important part of the diagnostic work up of chronic liver disease, as it directly affects prognosis and treatment as well as determining potential for reversibility. Liver biopsy is the standard method to stage fibrosis and allows grading of hepatic steatosis, necrosis, and inflammatory activity. There are several liver fibrosis staging systems, though the most often used is the METAVIR system (F0-F1: no to minimal fibrosis, F2-3: moderate to severe fibro- sis, F4: cirrhosis). The most advanced stage of fibrosis is cirrhosis (METAVIR F4), which is strongly associated with liver-induced morbidity and mortality (1). Therefore, identification of patients with liver fibrosis in the early reversible stages of the disease is imperative. However, biopsy is invasive and may be associated with complications, including hemorrhage, infection, arteriovenous fistulazition and sedation-related complications (2, 3). Given these risks and the importance of early detection, ultrasound elastography has been investigated as a noninvasive technique to grade liver fibrosis.

Ultrasound elastography is a technique which uses a modified ultrasound device to propagate elastic shear waves in the liver and measure liver stiffness or elasticity of the tis- 
sue. Increased shear wave velocity indicates decreased liver elasticity and greater hepatic disease (4). Abnormal hepatic ultrasound elastography (UE) has been demonstrated in patients with biliary atresia, cystic fibrosis (CF), non-alcoholic fatty liver disease (NAFLD), non-alcoholic steatohepatitis (NASH) and autoimmune liver disease (57). Shear wave velocity has been shown to correlate with the degree of fibrosis found in children undergoing liver biopsy (4, 7-9). In adults, UE has been used for staging of chronic liver disease in the setting of chronic viral hepatitis and NAFLD/NASH (10).

We will discuss the technical aspects of the various $\mathrm{UE}$ techniques and review the elastography findings in several specific etiologies of chronic pediatric liver disease.

\section{Technical aspects of liver elastography}

The three main ultrasound-based non-invasive methods to evaluate liver stiffness are transient elastography (TE), point quantifi- cation shear wave elastography (pSWE) and two-dimensional shear wave elastography (2D SWE). The pSWE and 2D SWE techniques utilize acoustic radiation force impulse (ARFI) to generate the shear wave and image the liver. The patient should fast for 4-6 hours prior to the examination as the fibrotic liver may show falsely increased liver stiffness values in the nonfasting state (11). Patient should be imaged in the supine or slightly left lateral decubitus position with the right arm overhead and the probe placed in an intercostal position. The ARFI should be perpendicular to the liver capsule to prevent reverberation artifact below Glisson's capsule and pulsatility from near-by vessels, which can falsely elevate measured shear-wave speed (12). Multiple studies have shown increased accuracy in children with use of the pediatric $S$ probe transducer (13). The $S$ probe, as compared to the $\mathrm{M}$ probe, was developed for narrower intercostal spaces and smaller livers and uses a higher frequency of $5 \mathrm{MHz}$ as compared to the $\mathrm{M}$ probe which is larger and

\begin{tabular}{|c|c|c|c|}
\hline \multirow{2}{*}{ Parameter } & \multicolumn{3}{|l|}{ Ultrasound elastography techniques } \\
\hline & $\mathrm{TE}$ & pSWE & 2D SWE \\
\hline Advantages & $\begin{array}{l}\text { Clinical use more widely studied, } \\
\text { can be performed by clinician } \\
\text { without need for radiology, } \\
\text { inexpensive study }\end{array}$ & $\begin{array}{l}\text { Can be performed as an } \\
\text { independent procedure } \\
\text { or as add-on during } \\
\text { liver ultrasound, direct } \\
\text { visualization of the liver, } \\
\text { more accurate than TE in the } \\
\text { setting of chronic hepatitis C }\end{array}$ & $\begin{array}{l}\text { Can be performed as an } \\
\text { independent procedure or as add- } \\
\text { on during liver ultrasound, direct } \\
\text { visualization of the liver, more } \\
\text { accurate than TE in the setting } \\
\text { of chronic hepatitis C, provides } \\
\text { color display of larger ROI }\end{array}$ \\
\hline $\begin{array}{l}\text { Frequency for shear } \\
\text { wave generation }\end{array}$ & $40-50 \mathrm{~Hz}$, probe dependent & $100-500 \mathrm{~Hz}$ & $100-500 \mathrm{~Hz}$ \\
\hline Limitations & $\begin{array}{l}\text { Requires a dedicated machine, } \\
\text { limited by ascites, obesity and } \\
\text { narrow intercostal space. No } \\
\text { grayscale image of liver and less } \\
\text { accurate than ARFI techniques in } \\
\text { the setting of chronic hepatitis C }\end{array}$ & $\begin{array}{l}\text { Less information available on } \\
\text { clinical utility due to shorter } \\
\text { time in use }\end{array}$ & $\begin{array}{l}\text { Less information available on } \\
\text { clinical utility due to shorter time } \\
\text { in use }\end{array}$ \\
\hline $\begin{array}{l}\text { Measurement } \\
\text { location }\end{array}$ & $\begin{array}{l}\text { Right } 9^{\text {th }}-11^{\text {th }} \\
\text { intercostal space }\end{array}$ & $\begin{array}{l}\text { Hepatic segment } \\
\text { VII or VIII }\end{array}$ & $\begin{array}{l}\text { Hepatic segment } \\
\text { VII or VIII }\end{array}$ \\
\hline $\begin{array}{l}\text { Region of interest } \\
\text { size }\end{array}$ & $\begin{array}{l}\text { Approximately } \\
4 \mathrm{~cm}^{3}\end{array}$ & $\begin{array}{l}\text { Approximately } \\
0.5-1 \mathrm{~cm}^{3}\end{array}$ & $\begin{array}{l}\text { Approximately } \\
20 \mathrm{~cm}^{3}\end{array}$ \\
\hline
\end{tabular}

$\mathrm{TE}=$ transient elastography; $\mathrm{pSWE}=$ point quantification shear wave elastography; $2 \mathrm{D}$ SWE=two-dimensional shear wave elastography; ROI=region of interest. 
operates at $3.5 \mathrm{MHz}$. The greatest accuracy of stiffness results from multiple measurements in the same location during a breath hold of a few seconds (14). For all techniques the literature recommends obtaining 10 measurements and reporting the median value, the majority of which should have a numerical result and not " 0.00 ", making them invalid $(12,15)$. Lastly, the interquartile ratio (IQR) can be used to evaluate the variability of measurements and should be less than 0.30. TE is an older technique than pSWE and 2D SWE, and therefore more literature is available concerning its results and clinical use. TE differs from pSWE and 2D SWE because it does not utilize direct image visualization (Table 1).

\section{Transient elastography technique}

The TE technique utilizes sound waves to measure liver stiffness, but no anatomic ultrasound images are generated. A $5 \mathrm{MHz}$ probe is used for children and is typically placed over the right liver at the 9th-11th intercostal space and a portion of liver $6 \mathrm{~cm}$ deep is examined. The TE provides a schematic charting the shear wave as a function of time and amplitude within the region of interest (ROI). The ROI is about $4 \mathrm{~cm} 3$. For the measurement to be considered valid there must be at least 10 values with a ratio of valid measurements to the total number of measurements equaling at least $60 \%$ and an IQR of less than $30 \%$ of the median liver stiffness value (IQR/median liver stiffness measurements $</=30 \%)(16)$. The value is expressed in kilopascals $(\mathrm{kPa})$.

The TE approach is appealing for its wide availability and ease of use by referring clinicians. However, the lack of image guidance to direct the ROI choice and avoid vessels is a drawback. Other factors that may limit evaluation by TE are the presence of ascites, obesity and narrow intercostal space. Lastly, while there is more available literature on TE, recent studies have shown that techniques which utilize ARFI are more accurate in assessing significant fibrosis in the setting of chronic hepatitis $\mathrm{C}(12,17)$.

\section{Point quantification shear wave elastography technique}

The pSWE technique uses ARFI technology and measures liver tissue displacement within a small ROI (about 0.5-1 cm3) in response to the generated shear waves. The shear wave speed through the liver tissue can then be used to calculate liver stiffness. The B-mode imaging allows the user to visualize the liver parenchyma, choose different portions of the liver to sample and avoid vessels or masses while measuring shear wave speed $(12,14)$ (Fig. 1). The shear wave speed is measured in meters per second but can be converted to $\mathrm{kPa}$.

\section{Two-dimensional shear wave elastography technique}

The 2D SWE technique also uses ARFI technology to image the liver, using a large ROI of approximately $20 \mathrm{~cm} 3$. It also allows the user to view the elastographic measures in color display as they are gathered. The shear wave speed is measured in meters per second but can be converted to $\mathrm{kPa}$. Again, the user can visualize the liver parenchyma in real time and avoid vessels and masses while measuring shear wave speed (14).

\section{Utility in clinical practice}

There are many potential clinical indications for the use of elastography in the evaluation of pediatric liver disease. Commonly, the objective is to assess patients with chronic liver disease and identify liver fibrosis in its early stages to help direct treatment. UE has been 


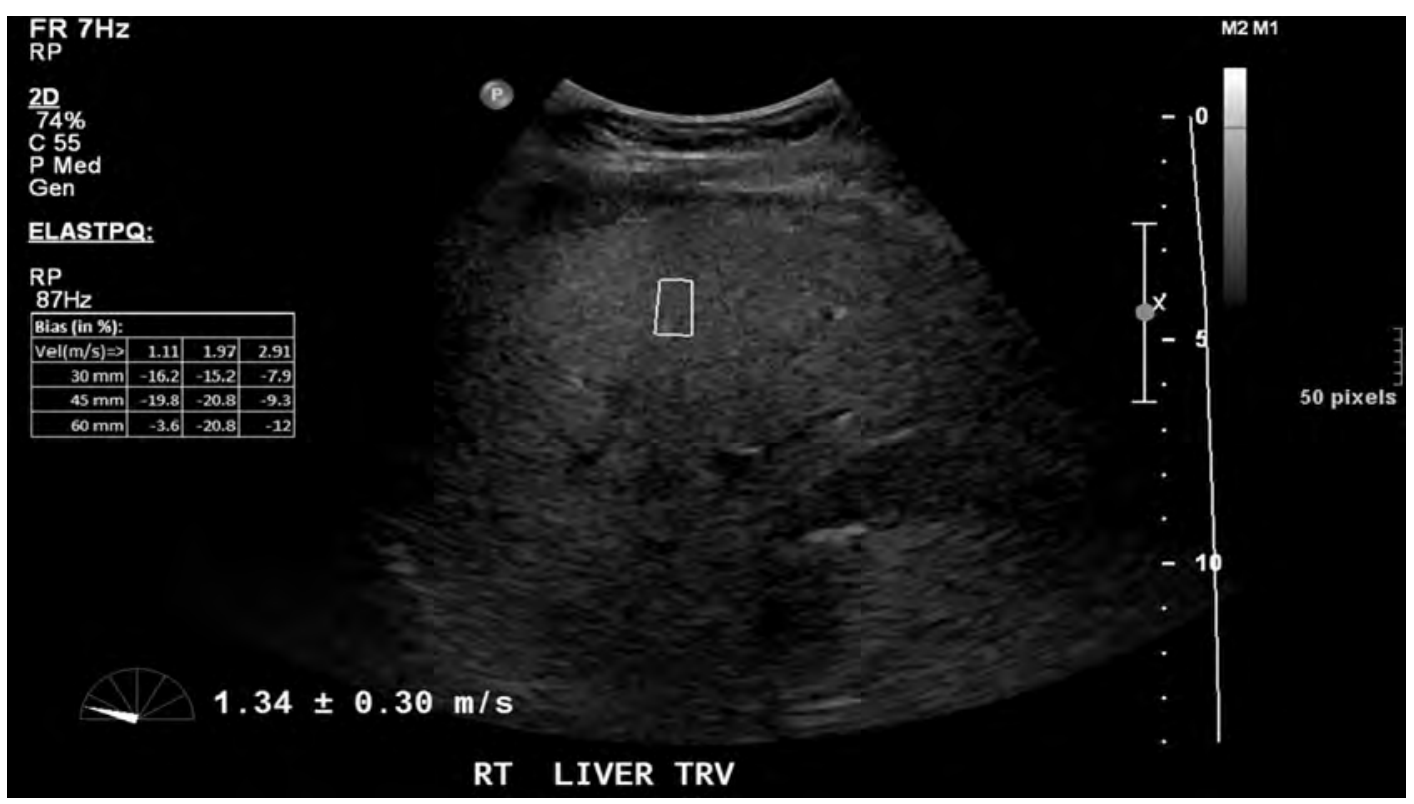

Fig. 1 Point quantification shear wave elastography image in a 10-year-old female with history of hepatic steatosis. Measurement is obtained from the region of interest denoted by the white box. A total of ten measurements are obtained from the same location.

investigated in pediatric patients with several liver diseases, including NAFLD, chronic viral hepatitis, CF, biliary atresia and liver transplant. Its potential role in each of these diseases will be discussed.

\section{Non-alcoholic fatty liver disease}

NAFLD is the most common chronic liver disease in children and is strongly associated with childhood obesity, metabolic syndrome and insulin resistance. The more aggressive and inflammatory form of NAFLD/ $\mathrm{NASH}$ as well as fibrosis and cirrhosis can develop during childhood (18). Children with NAFLD were found to have a significant correlation between shear wave velocity using ARFI ultrasound elastography and aspartate aminotransferase (AST) levels. In those patients with hepatic steatosis the shear wave velocity was found to be higher than in the healthy control group, indicating that fatty deposition increases liver stiffness (9). Wong compared TE with liver biopsy in
246 NAFLD adult patients and found 91\% sensitivity and $75 \%$ specificity for predicting severe fibrosis. Discordance between TE and liver biopsy was seen in approximately 13\% of cases and was associated with insufficient tissue sampling during biopsy and early stage fibrosis (19). TE was determined to be a useful screening mechanism for advanced liver fibrosis in the setting of NAFLD. Another study found TE to be accurate in identifying children without significant fibrosis as well as those with advanced fibrosis, as compared to biopsy. A limitation observed during this study was failure to obtain valid TE measurements in obese children with body mass in$\operatorname{dex}(\mathrm{BMI})>l=35(20)$.

\section{Chronic viral hepatitis}

The sequelae of viral hepatitis in those patients who do not clear the virus are serious and can result in long term morbidity and mortality from fibrosis, cirrhosis and development of HCC. Hepatitis B is most often 
acquired postnatally in children, with under $4 \%$ going on to develop cirrhosis or HCC. Hepatitis $\mathrm{C}$ is most commonly acquired perinatally, with $10-20 \%$ clearance rate before 2 years old. The majority of patients who do not clear the virus before the first 2 years of life go on to develop chronic hepatitis (21). Liver shear wave velocity was found to be higher in children with chronic viral hepatitis and correlated with more advanced histologic degree of fibrosis (9). Studies show that TE can differentiate normal liver and mild fibrosis from significant fibrosis and cirrhosis, which is important for the clinical management of patients and their treatment. TE is less accurate in distinguishing between mild, moderate and severe degrees of liver fibrosis (22-24). TE has been found to be a more accurate prognostic indicator than liver biopsy in adults with chronic hepatitis C: those with higher stage liver fibrosis had a significantly decreased 5-year survival as compared to those with lower stage fibrosis (25). Liver stiffness measured with TE correlated with the risk of developing HCC in adults with hepatitis B and C $(26,27)$. The European Association for the Study of the Liver has recommended the use of TE in the management of chronic viral hepatitis in adults (28).

\section{Cystic fibrosis}

Ten percent of patients with CF may present with severe liver disease. Patients with severe cystic fibrosis-related liver disease (CFLD) usually present in childhood and may suffer complications such as malnutrition, portal hypertension and variceal hemorrhage, and liver failure. In its most severe form biliary cirrhosis can develop rapidly (29). CFLD may show patchy fibrotic changes early on in the disease process, so liver biopsy may miss a diseased region due to sampling error. Witters et al. (30) evaluated sixty-six pediatric cystic fibrosis patients with TE and compared them to a control group of healthy children. Liver disease was defined clinically based on the presence of hepatomegaly or splenomegaly and biochemically based on elevated liver function tests (1.5 times the upper limit of normal for age) for 3-6 months. Ultrasound findings were collected for these patients and scored based on the appearance of hepatic parenchyma, liver edge nodularity and presence and severity of periportal fibrosis and liver stiffness was measured utilizing TE. The authors found liver stiffness to be significantly elevated in patients with clinical CFLD, biochemical CFLD and ultrasonographic CFLD. The TE was comparable to the ultrasound scoring system in evaluating for CFLD, sensitivity of $63 \%$ and specificity of $87 \%$. TE was also comparable to the ultrasound scoring system in detection of hepatic fibrosis (30). Similarly, another study found that elasticity values of controls, pancreatic sufficient CF patients and pancreatic insufficient CF patients with an ultrasound showing either normal or homogenously hyperechoic livers, were comparable and significantly lower than in CF patients with heterogenous, nodular livers or with evidence of portal hypertension on ultrasound $(\mathrm{P}<0.002)$. Interestingly, it was found that male adults and male children with CF had significantly higher liver stiffness than females in the same group $(\mathrm{P}=0.0013)(31)$.

\section{Biliary atresia}

Biliary atresia is characterized by inflammatory obliterative cholangiopathy of the extrahepatic biliary tree and presents with biliary obstruction. The most common cause of biliary atresia is idiopathic and often presents in the neonatal period with jaundice and pale stools. Biliary atresia is a surgical emergency and requires timely surgical intervention. If left untreated biliary atresia can progress to hepatic fibrosis and cirrhosis. Traditionally, 
ultrasound is used to evaluate for biliary atresia looking for indicators of disease such as absent or irregular gallbladder, absent common bile duct and presence of an echogenic triangular cord above the porta hepatis. Leschied et al. (32) found that pSWE can differentiate biliary atresia from other neonatal liver diseases that have a similar clinical presentation such as neonatal hepatitis and Alagille syndrome. The mean shear wave speed was found to be significantly higher in children with biliary atresia as compared to those with other causes of cholestasis. The pSWE technique found a mean velocity of $2.08 \mathrm{~m} / \mathrm{s}$ in biliary atresia compared to $1.28 \mathrm{~m} / \mathrm{s}$ in other causes of cholestasis, and the 2D SWE technique found a mean velocity of $3.14 \mathrm{~m} / \mathrm{s}$ in biliary atresia compared to $1.61 \mathrm{~m} / \mathrm{s}$ in other causes of cholestasis (32). TE significantly correlates with histologic fibrosis staging in children with biliary atresia. One study revealed that TE, when compared to liver biopsy, accurately diagnosed cirrhosis in $90 \%$ of patients. However, while TE demonstrated a high degree of accuracy in distinguishing cirrhosis from non-cirrhosis, it was less accurate in differentiating the stage of fibrosis in non-cirrhotic patients (33). A TE value of greater than $9.6 \mathrm{kPa}$ in biliary atresia had a sensitivity of $89.5 \%$ and specificity of $75 \%$ for severe fibrosis and a TE value greater than $18.1 \mathrm{kPa}$ was $100 \%$ sensitive and $90.5 \%$ specific for cirrhosis (13).

\section{Liver transplant}

Goldschmidt et al. (34) showed a good correlation between TE and histologic degree of fibrosis in pediatric patients post liver transplant. They also found liver stiffness values for transplanted children without fibrosis to be significantly higher than healthy controls. It is suggested that TE be used as a mechanism for follow-up after establishing a baseline for an individual patient (6). One study which used TE to evaluate post-transplant livers in adults infected with hepatitis $\mathrm{C}$ showed areas under the receiver operator curve (AUROC) of .94 and .90 (where AUROC of 1.0 is characteristic of an ideal test and 0.5 is non-diagnostic) for identifying significant fibrosis, defined as METAVIR stages $>l=2$ (35). TE is an accurate non-invasive method to detect significant fibrosis as well as the absence of fibrosis in liver transplant.

\section{Conclusion}

Ground-breaking research over the past several years has shown ultrasound elastography to be a potentially useful tool in the management of chronic liver disease which may provide a noninvasive method to screen for liver fibrosis in the future. TE has been established as an accurate measure of liver fibrosis in NAFLD, making it a potentially useful screening test for advanced liver fibrosis in patients with fatty liver disease. In the setting of viral hepatitis, TE can differentiate normal liver and mild fibrosis from significant fibrosis and cirrhosis. TE may also be used as a prognostic indicator, as liver stiffness has been correlated with the risk of developing HCC in adults with hepatitis B and C. SWE can help differentiate biliary atresia from other neonatal liver diseases and TE significantly correlates with histologic fibrosis staging in children with biliary atresia. There is good correlation of TE with histologic degree of fibrosis in pediatric patients post liver transplant and it has been shown as an accurate, non-invasive method to detect significant fibrosis as well as the absence of fibrosis.

While not widely available in clinical practice yet, this technology is available in newer ultrasound machine models and is expected to become more widely available as facilities update their devices and clinicians request this information. At our institution currently, ultrasound elastrography is being 
utilized as a research tool and questions remain as this new technology transitions from a research method to a clinical tool. The role of ultrasound elastography in the imaging algorithm for pediatric patients with chronic liver disease must be elucidated. Normative values and appropriate intervals for testing must be established. As obesity and liver disease often go hand in hand, the accuracy of ultrasound elastography must be defined in this population in whom TE is known to be less accurate. The role of ultrasound elastography in screening patients at high risk for developing HCC must be defined.

Potential indications for elastography are increasing with the growing fund of knowledge gained from continued investigation into this burgeoning modality. We have examined multiple disease entities in which studies have shown ultrasound elastography to be useful in assessing extent, progression and prognosis of pediatric liver disease. $\mathrm{Fa}$ miliarity with this new non-invasive technique will be imperative in upcoming years as it moves from research into clinical practice.

Authors' contributions: Conception and design: IHS, MCL; Acquisition, analysis and interpretation of data: IHS, MCL; Drafting the article: IHS, MCL; Revising it critically for important intellectual content: IHS, MCL.

Conflict of interest: The authors declare that they have no conflict of interest.

\section{References}

1. Younossi ZM, Stepanova M, Rafiq N, Makhlouf H, Younoszai Z, Agrawal R, Goodman Z. Pathologic criteria for nonalcoholic steatohepatitis: interprotocol agreement and ability to predict liver-related mortality. Hepatology. 2011;53(6):1874-82.

2. Seeff LB, Everson GT, Morgan TR, Curto TM, Lee WM, Ghany MG, et al.; HALT-C Trial Group. Complication rate of percutaneous liver biopsies among persons with advanced chronic liver disease in the HALT-C trial. Clin Gastroenterol Hepatol. 2010;8(10):877-83.
3. Stotland BR, Lichtenstein GR. Liver biopsy complications and routine ultrasound. Am J Gastroenterol. 1996;91(7):1295-6.

4. Dillman JR, Heider A, Bilhartz JL, Smith EA, Keshavarzi N, Rubin JM, et al. Ultrasound shear wave speed measurements correlate with liver fibrosis in children. Pediatr Radiol. 2015p;45(10):1480-8.

5. Dehghani SM, Imanieh MH, Haghighat M, Malekpour A, Falizkar Z. Etiology and complications of liver cirrhosis in children:report of a single center from southern iran. Middle East J Dig Dis. 2013;5(1):41-6.

6. Goss JA, Shackleton CR, McDiarmid SV, Maggard M, Swenson K, Seu P, et al. Long-term results of pediatric liver transplantation: an analysis of 569 transplants. Ann Surg. 1998;228(3):411-20.

7. Keller PD, Nute WL Jr. Cirrhosis of the liver in children; a clinical and pathologic study of 40 cases. J Pediatr. 1949;34(5):588-615.

8. Hanquinet S, Rougemont AL, Courvoisier D, Rubbia-Brandt L, McLin V, Tempia M, Anooshiravani M. Acoustic radiation force impulse (ARFI) elastography for the noninvasive diagnosis of liver fibrosis in children. Pediatr Radiol. 2013;43(5):545-51.

9. Marginean CO, Marginean C. Elastographic assessment of liver fibrosis in children: A prospective single center experience. Eur J Radiol. 2012;81(8):e870-4.

10. Ferraioli G, Filice C, Castera L, Choi BI, Sporea I, Wilson SR, et al. WFUMB guidelines and recommendations for clinical use of ultrasoundelastography: Part 3: liver. Ultrasound Med Biol. 2015;41(5):1161-79.

11. Mederacke I, Wursthorn K, Kirschner J, Rifai $\mathrm{K}$, Manns MP, Wedemeyer $\mathrm{H}$, et al. Food intake increases liver stiffness in patients with chronic or resolved hepatitis $\mathrm{C}$ virus infection. Liver Int. 2009;29(10):1500-6.

12. Ferraioli G, Tinelli C, Dal Bello B, Zicchetti M, Filice G, Filice C; Liver Fibrosis Study Group. Accuracy of real-time shear wave elastography for assessing liver fibrosis in chronic hepatitis C: a pilot study. Hepatology. 2012;56(6):2125-33.

13. Shin NY, Kim MJ, Lee MJ, Han SJ, Koh H, Namgung R, Park YN. Transient elastography and sonography for prediction of liver fibrosis in infants with biliary atresia. J Ultrasound Med. 2014;33(5):853-64.

14. Barr RG, Ferraioli G, Palmeri ML, Goodman ZD, Garcia-Tsao G, Rubin J, et al. Elastography Assess- 
ment of Liver Fibrosis: Society of Radiologists in Ultrasound Consensus Conference Statement. Radiology. 2015;276(3):845-61.

15. Procopet B, Berzigotti A, Abraldes JG, Turon F, Hernandez-Gea V, García-Pagán JC, et al. Realtime shear-wave elastography: applicability, reliability and ccuracy for clinically significant portal hypertension. J Hepatol. 2015;62(5):1068-75.

16. Castera L, Forns X, Alberti A. Non-invasive evaluation of liver fibrosis using transient elastography. J Hepatol. 2008;48(5):835-47.

17. Bavu E, Gennisson JL, Couade M, Bercoff J, Mallet V, Fink M, et al. Noninvasive in vivo liver fibrosis evaluation using supersonic shear imaging: a clinical study on 113 hepatitis $C$ virus patients. Ultrasound Med Biol. 2011;37(9):1361-73.

18. Alisi A, Feldstein AE, Villani A, Raponi M, Nobili V. Pediatric nonalcoholic fatty liver disease: a multidisciplinary approach. Nat Rev Gastroenterol Hepatol. 2012;9(3):152-61.

19. Wong VW, Vergniol J, Wong GL, Foucher J, Chan HL, Le Bail B, et al. Diagnosis of fibrosis and cirrhosis using liver stiffness measurement in nonalcoholic fatty liver disease. Hepatology. 2010;51(2):454-62.

20. Nobili V, Vizzutti F, Arena U, Abraldes JG, Marra F, Pietrobattista A, Fruhwirth R, Marcellini M, Pinzani M. Accuracy and reproducibility of transient elastography for the diagnosis of fibrosis in pediatric nonalcoholic steatohepatitis. Hepatology. 2008;48(2):442-8.

21. European Paediatric Hepatitis C Virus Network. Three broad modalities in the natural history of vertically acquired hepatitis $C$ virus infection. Clin Infect Dis. 2005;41(1):45-51.

22. Talwalkar JA, Kurtz DM, Schoenleber SJ, West CP, Montori VM. Ultrasound-based transient elastography for the detection of hepatic fibrosis: systematic review and meta-analysis. Clin Gastroenterol Hepatol. 2007;5(10):1214-20.

23. Friedrich-Rust M, Ong MF, Martens S, Sarrazin C, Bojunga J, Zeuzem S, et al. Performance of transient elastography for the staging of liver fibrosis: a meta-analysis. Gastroenterology. 2008;134(4):96074.

24. Tsochatzis EA, Gurusamy KS, Ntaoula S, Cholongitas E, Davidson BR, Burroughs AK. Elastography for the diagnosis of severity of fibrosis in chronic liver disease: a meta-analysis of diagnostic accuracy. J Hepatol. 2011;54(4):650-9.
25. Vergniol J, Foucher J, Terrebonne E, Bernard PH, le Bail B, Merrouche W, et al. Noninvasive tests for fibrosis and liver stiffness predict 5-year outcomes of patients with chronic hepatitis C. Gastroenterology. 2011;140(7):1970-9, 1979.e1-3.

26. Masuzaki R, Tateishi R, Yoshida H, Goto E, Sato T, Ohki T, et al. Prospective risk assessment for hepatocellular carcinoma development in patients with chronic hepatitis $\mathrm{C}$ by transient elastography. Hepatology. 2009;49(6):1954-61.

27. Jung KS, Kim SU, Ahn SH, Park YN, Kim do Y, Park JY, Chon CY, et al. Risk assessment of hepatitis B virus-related hepatocellular carcinoma development using liver stiffness measurement (FibroScan). Hepatology. 2011;53(3):885-94.

28. European Association for the Study of the Liver. EASL Clinical Practice Guidelines: management of hepatitis C virus infection. J Hepatol. 2011;55(2):245-64.

29. Cystic Fibrosis Foundation. Patient Registry 2011 Annual Data Report. Bethesda, MD: Cystic Fibrosis Foundation; 2012

30. Witters P, De Boeck K, Dupont L, Proesmans M, Vermeulen F, Servaes R, et al. Non-invasive liver elastography (Fibroscan) for detection of cystic fibrosis-associated liver disease. J Cyst Fibros. 2009;8(6):392-9.

31. Menten R, Leonard A, Clapuyt P, Vincke P, Nicolae AC, Lebecque P. Transient elastography in patients with cystic fibrosis. Pediatr Radiol. 2010;40(7):1231-5.

32. Leschied JR, Dillman JR, Bilhartz J, Heider A, Smith EA, Lopez MJ. Shear wave elastography helps differentiate biliary atresia from other neonatal/infantile liver diseases. Pediatr Radiol. 2015;45(3):366-75.

33. Shen QL, Chen YJ, Wang ZM, Zhang TC, Pang WB, Shu J, et al. Assessment of liver fibrosis by Fibroscan as compared to liver biopsy in biliary atresia. World J Gastroenterol. 2015;21(22):6931-6.

34. Goldschmidt I, Stieghorst H, Munteanu M, Poynard T, Schlue J, Streckenbach C, Baumann U. The use of transient elastography and non-invasive serum markers of fibrosis in pediatric liver transplant recipients. Pediatr Transplant. 2013;17(6):525-34.

35. Corradi F, Piscaglia F, Flori S, D'Errico-Grigioni A, Vasuri F, Tamé MR, et al.; Bologna Liver Transplantation Group. Assessment of liver fibrosis in transplant recipients with recurrent $\mathrm{HCV}$ infection: usefulness of transient elastography. Dig Liver Dis. 2009; 41(3):217-25. 